\title{
Characterization of the extent of a large outbreak of Legionnaires' disease by serological assays
}

Øystein Simonsen ${ }^{1 *}$, Elisabeth Wedege ${ }^{2}$, Anita Kanestrøm³ ${ }^{3}$ Karin Bolstad², Ingeborg S Aaberge ${ }^{2}$, Eivind Ragnhildstveit ${ }^{3}$ and Jetmund Ringstad ${ }^{1}$

\begin{abstract}
Background: In May 2005, a long-distance outbreak of Legionnaires' disease (LD) caused by Legionella pneumophila serogroup 1 occurred in south-east Norway. The initial outbreak investigation without serology identified 56 laboratory-confirmed LD cases of whom 10 died. However, 116 patients with community-acquired pneumonia might belong to the outbreak based on epidemiological investigations, but acute laboratory tests other than serology were negative or not performed. To assess the true extent of the outbreak, we evaluated two serological assays in order to reclassify the 116 patients with indeterminate case status.

Methods: Two polyvalent antibody tests, a serogroup 1-6 immunofluorescence assay (IFA) and a serogroup 1-7 enzyme-linked immunosorbent assay (ELISA) were used. They were evaluated with cases defined as culture- or urinary antigen positive LD patients $(n=40)$ and non-cases defined as confirmed non-LD patients $(n=39)$ and healthy control subjects $(n=62)$. The 116 patients, who were negative in culture, polymerase chain reaction and/or urinary antigen tests, were analysed by the same serological assays. Antibodies to the outbreak strain were determined by immunoblotting.

Results: In the evaluation study, the sensitivity and specificity of a $\geq 4$-fold IFA titre change was 38\% and 100\%, respectively, with corresponding values of $30 \%$ and $99 \%$ for seroconversion in ELISA. A single high positive IFA titre yielded sensitivity and specificity of $73 \%$ and $97 \%$, respectively, with corresponding values of $68 \%$ and $96 \%$ for a single high immunoglobulin (lg) $\mathrm{G}$ and/or IgM in ELISA.

Based on this evaluation, the following serological testing identified 47 more LD cases, and the outbreak thus comprised 103 cases with a case fatality rate of 10\%. About the same proportion (70\%) of the urinary antigen positive and negative LD cases had antibodies to the serogroup-specific lipopolysaccharide of the outbreak strain. In addition to the 103 LD cases, Legionella infection could not be verified or excluded in 32 patients based on epidemiology and/or lack of microbiological sampling.
\end{abstract}

Conclusions: The acute-phase tests (culture, polymerase chain reaction, and urinary antigen) identified less than 55\% of the 103 patients in this outbreak. Serological testing thus remains an important supplement for diagnosis of LD and for determination of outbreak cases.

Keywords: Legionella pneumophila, Outbreak, Serological assays, Community-acquired pneumonia

\footnotetext{
* Correspondence: oystein.simonsen@so-hf.no

'Department of Medicine, Østfold Hospital Trust, Fredrikstad, Norway

Full list of author information is available at the end of the article
} 


\section{Background}

Legionnaires' disease (LD) has been considered a rare cause of community-acquired pneumonia (CAP) in Norway. The last 10 years, about 6 cases per million inhabitants were reported annually [1] which is lower than the incidence rate of more than 10 per million reported in overall Europe [2]. However, a study in 2005 showed considerable underreporting of the disease in Norway [3]. Insufficient use of diagnostic testing and false negative diagnostic tests may lead to underreporting in passive surveillance systems. A disease incidence of almost 80 per million was estimated in a population-based study [4], and Legionella spp. cause between $2 \%$ and $16 \%$ of CAP cases in prospective studies [5-7], rendering Legionella spp. one of the most common pathogens in CAP. $L$. pneumophila serogroup 1 (Lp 1) is responsible for $70 \%$ to $90 \%$ of all culture positive LD cases $[8,9]$.

Microbiological diagnosis of LD during acute illness is based on culture, polymerase chain reaction (PCR) of respiratory samples, and/or detection of Legionella antigen in urine. Isolation of Legionella spp. by culture is considered the gold standard for diagnosing LD, but the sensitivity is low. PCR-based methods are rapid and more sensitive than culture [10], but more experience in clinical use is needed [11]. The urinary antigen test (UAT) has become the most performed test in diagnosing LD [8] because of its easy performance and rapidity combined with a reasonable sensitivity ranging from $40 \%$ to $94 \%$ [12-14]. However, it is only reliable for Lp 1 infections. Serological tests rarely allow a diagnosis during the acute phase and are regarded more useful for epidemiological purposes.

In May 2005, an outbreak of LD caused by Lp 1 (ST15, monoclonal subgroup Benidorm [15]) occurred in southeast Norway [16]. The source was identified as an industrial air scrubber with a long-distance spread of more than $10 \mathrm{~km}$ [16]. In addition, the river Glomma has been proposed as a disseminator due to the release of waste water from industrial aeration ponds [17]. The initial outbreak investigation identified 56 patients diagnosed with LD by a positive Lp 1 culture, PCR, and/or UAT [16], but serological testing was not performed. As the aim of that study was mainly to identify the source of the outbreak, probable cases were not included. In the present investigation, sera from the laboratory confirmed LD cases were used to evaluate two commercial serological assays against L. pneumophila, which previously had been evaluated in one study only [18]. Based on the sensitivities and specificities obtained in our evaluation, the tests were then employed to measure antibody levels in sera from all CAP patients, who were referred to the local hospital during the outbreak. Our aims were to diagnose additional LD cases and so to determine the extent of the outbreak.

\section{Methods}

\section{Setting}

Østfold Hospital Trust is an acute care hospital that serves the 260,000 inhabitants of Østfold County, including the 120,000 inhabitants of the twin cities Sarpsborg and Fredrikstad. The regional epidemiology of CAP has been constant in May/June during the years 2001 - 2004 with an average of 16 referrals per week ( 2.3 per day) to the hospital. On 21 May 2005, an LD outbreak was suspected, and the initial outbreak investigation identified 56 cases of LD (53 cases admitted to our hospital and 3 cases to other hospitals in Norway), confirmed by either a positive Legionella UAT and/or culture of Lp 1 and/or PCR [16]. In order to identify potentially undiagnosed cases, adult patients referred to our hospital with radiographically confirmed CAP during a five-week period from 9 May to 12 June 2005 were invited to a serological study. The period chosen was based on the previous report of this outbreak [16]. In addition, healthy controls, recruited from the hospital staff, were invited in late May for evaluation of the serological assays. The study was approved by the Regional Committee for Medical and Health Research Ethics (REC) South East, P.O BOX 1130, Blindern, NO 0318 Oslo, Norway. Written informed consent for participation in the study was obtained from all participants.

\section{Microbiological investigation}

Blood cultures were drawn from CAP patients referred to the emergency department during the five-week period, and sputum cultures were sampled from patients with expectoration. Isolated bacterial species were classified as an aetiological pathogen if predominant growth of a typical respiratory tract pathogen was observed. Lung tissue specimens from deceased patients and sputum were also analysed by a commercial PCR test (Onar LpQP; Minerva Biolabs $\mathrm{GmbH}$, Germany) for detection of Legionella spp. and cultured for Legionella (plated both directly and after an acid decontamination step onto selective and non-selective buffered charcoal-yeast extract agar). Isolates were serogrouped with Legionella Latex Test (Oxoid, Basingstoke, UK).

Urine samples from patients and healthy control subjects were analysed by the Now Legionella Urinary Antigen Test and the Now Streptococcus pneumoniae Urinary Antigen Test (Binax, Portland, Maine) for qualitative detection of soluble Legionella and S. pneumoniae antigen, respectively.

Sera from CAP patients were collected during the acute phase ( 0 - 15 days after symptom onset) if available, $4-6$ weeks, and 3 months after hospital admission. Sera from healthy employees were collected in late May and after approximately 1 and 3 months. All sera were stored at $-20^{\circ} \mathrm{C}$. Two commercial polyvalent serological 
L. pneumophila assays were used for evaluation of the methods and the outbreak investigation: a serogroup 1-6 immunoglobulin (Ig) G/IgM/IgA immunofluorescence assay (IFA) (Meridian Bioscience Europe, Milan, Italy) and a serogroup 1-7 enzyme-linked immunosorbent assay (ELISA) (Serion ELISA classic, Institut Virion/Serion $\mathrm{GmbH}$, Würzburg, Germany) with separate levels of IgG and IgM antibodies measured in an ELISA robot (DSX Automated System, Dynex Technologies, Inc, Virginia). The coefficients of variation for four standard samples per plate in this study were $7.6 \%$ for IgG (range 4.1 to 10.0 ) and $6.6 \%$ for IgM (range 5.8 to 7.4 ) compared with the maximal interserial coefficient of variation of $16 \%$ given by the manufacturer [19]. Both tests were performed according to the manufacturers' instructions and analysed without knowledge of Lp 1 infection status.

\section{Evaluation of the serological assays}

The reference standard for the serological evaluation was based on the EU case definition [20], but excluding serology as microbiological evidence of infection. Hence, patients with radiographically confirmed CAP admitted during the defined five-week period and with either isolation of L. pneumophila from a respiratory sample and/or a positive L. pneumophila UAT were defined as cases. Patients with confirmed non-LD pneumonia and healthy control subjects were considered to be non-cases. The definition of confirmed non-LD pneumonia was radiographically documented CAP and a negative Legionella UAT and culture with either a) proven aetiology other than Legionella, b) date of symptom onset before 10 May or after 27 May 2005 (based on the symptom onset period of the initial 56 cases), and/or c) residency outside and not visiting the outbreak area which was defined by the plume model from the aerosol dispersion investigation [16]. Only LD cases and healthy controls with paired serum samples were included in the evaluation study.

The assays were evaluated from the antibody responses corresponding to the EU laboratory criteria for confirmed and probable cases in addition to an alternative ELISAratio method:

1) Serologically confirmed LD was defined as a $\geq 4$-fold change in IFA titres in paired sera or seroconversion in ELISA to a positive $\operatorname{IgG}(\geq 50 \mathrm{U} / \mathrm{ml})$ or $\operatorname{IgM}(\geq 120 \mathrm{U} /$ $\mathrm{ml}$ ), which include the borderline ranges given by the manufacturer [19].

2) Serologically probable LD was defined as a single high or high standing antibody level with an IFA titre $\geq 128$, as given by the manufacturer, and/or ELISA IgG $\geq 50 \mathrm{U} / \mathrm{ml}$ and/or $\operatorname{IgM} \geq 120 \mathrm{U} / \mathrm{ml}$.

3) An alternative definition of probable LD was based on a relative change of IgG and/or IgM antibody levels in paired serum samples in ELISA and calculated for all paired serum samples including antibody levels below the manufacturer's cut-off. To exclude clinically irrelevant changes in the low antibody ranges, only sera with IgG or IgM levels above the 75th percentile of the noncases (IgG $16 \mathrm{U} / \mathrm{ml}$ and IgM $26 \mathrm{U} / \mathrm{ml}$, respectively), were considered for this calculation. In paired sera with antibody levels below these values, the ratio was set to 1.0, indicating no change.

\section{Outbreak investigation}

Sera from patients with CAP, who lived within or visited the outbreak area, but were negative in Legionella culture, PCR, and/or UAT, were analyzed with the serological assays. From their antibody responses, patients with positive serology were defined as confirmed or probable LD cases and included in the outbreak.

\section{Classification of pneumonia severity}

Pneumonia severity was classified by CRB-65 [21], a score derived by four criteria of severity obtained on admission: confusion, respiratory rate ( $>30$ per minute), low blood pressure (diastolic pressure $\leq 60 \mathrm{mmHg}$ or systolic pressure $\leq 90 \mathrm{mmHg}$ ), and age $\geq 65$ years. A CRB-65 score $\geq 2$ was considered to be moderate to severe pneumonia.

\section{Immunoblotting}

To study if the antibody responses, determined in ELISA and IFA with the polyvalent antigens, were directed to the serogroup-specific lipopolysaccharide (LPS) antigen of the Lp 1 outbreak strain [22], immunoblotting with whole-cell suspensions of this strain was performed as described previously [23] with detection of IgG and IgM binding on separate strips. From each patient, the serum sample (diluted 1:200) with the highest IgG or IgM antibody level in ELISA was used. Blotting was also performed with proteinase K (Qiagen Gmbh, Hilden, Germany) treated cells [24] from the outbreak strain and from an Lp 1 isolate of subgroup France/Allentown to study LPS cross-reactive antibodies. The LPS patterns of the strains were obtained by silver-staining of sodium dodecyl sulphate polyacrylamide gels [25] as well as by incubation of strips with an Lp 1 monoclonal antibody from the Dresden panel [26]. Antibody binding to the corresponding LPS region on the strips was recorded visually as strong, weak, or no response.

\section{Statistical methods}

Statistical analyses were performed with SPSS version 16.0. Comparisons between groups were performed using the $\chi^{2}$ test or independent $\mathrm{t}$-test when appropriate. All tests were two-sided, and the significance level was set at 0.05 . Diagnostic sensitivity and specificity were calculated for each test using OpenEpi [27]. Correlation between the 
two serological assays was calculated by the Spearman rank order test.

\section{Results}

\section{Evaluation of the serological assays}

A total of 225 adult patients with CAP of any cause was referred to our hospital during the defined fiveweek period. Before serological testing was performed, LD and non-LD pneumonia were confirmed in 53 and 56 patients, respectively, leaving 116 patients with unknown aetiology (Figure 1). Non-LD was confirmed by the finding of another respiratory pathogen (16 patients), date of illness onset before 10 May or after 27 May (35 patients), and residency outside and not visiting the outbreak area (5 patients). The patients included for the serological assay evaluation comprised 40 LD cases (median age 68 years, range $35-94$ ) and 101 non-cases
(39 non-LD patients, median age 68 years, range $18-88$, and 62 healthy controls, median age 45 years, range $24-$ 62) from whom two or more sera were collected (Figure 1).

Sensitivities and specificities of the two serological assays for the different LD case definitions are shown in Table 1. The IFA and ELISA results were roughly equal for both the confirmed and probable LD case definitions, and combination of both assays resulted in somewhat better sensitivity without affecting the specificity. The two serological assays also demonstrated significant correlations between the IFA titres and the sum of IgG and IgM levels in ELISA with a Spearman rank correlation coefficient of $0.79(P<0.001)$.

In the alternative ELISA evaluation, the median IgGand IgM ratios were 1.7 (interquartile range (IQR) 1.1 2.7) and 3.1 (IQR $1.1-6.2$ ), respectively, for the LD

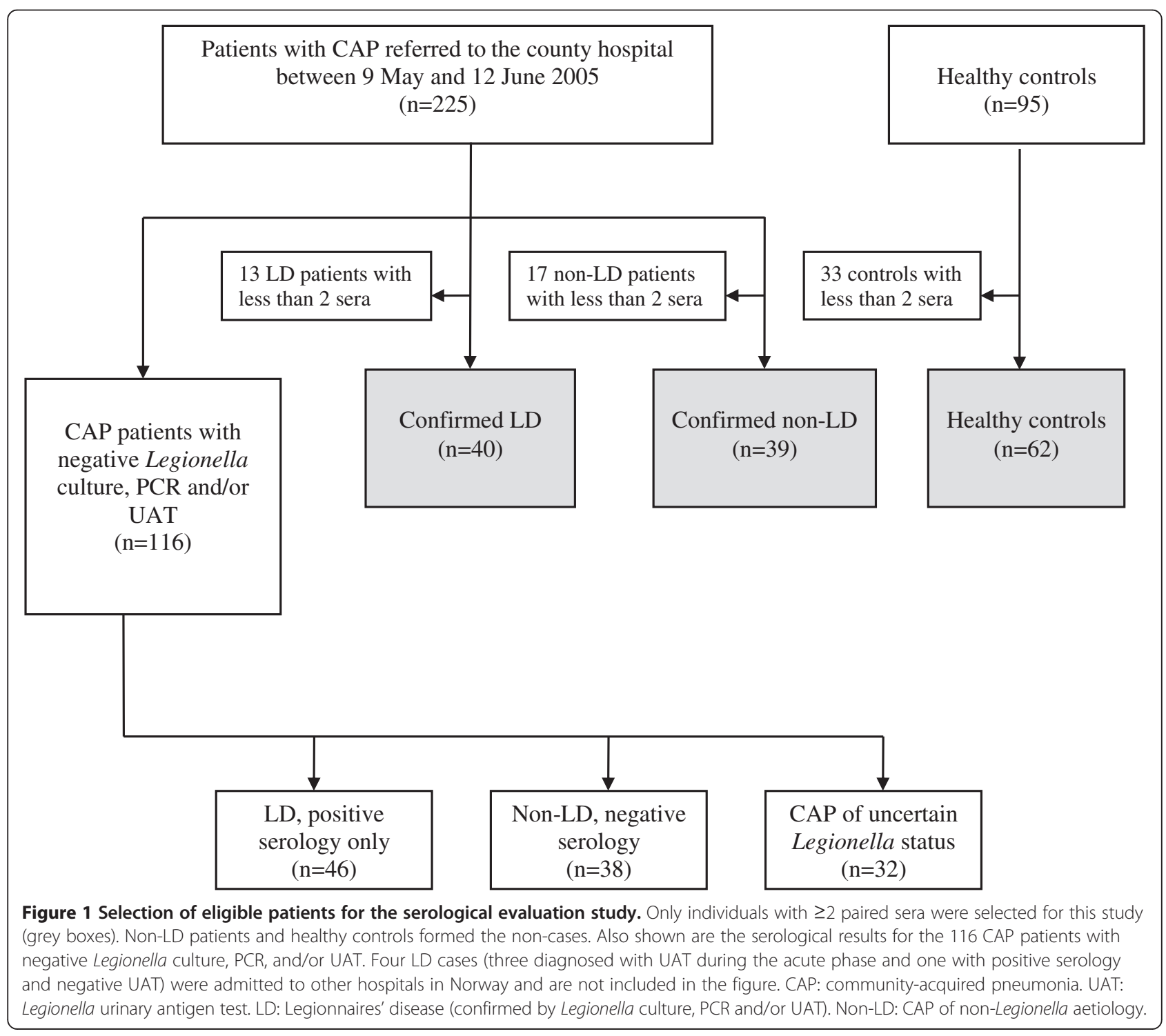


Table 1 Diagnostic sensitivity and specificity of IFA and ELISA tests for different Legionnaires' disease case definitions

\begin{tabular}{|c|c|c|c|}
\hline Case definition & Serological response & $\begin{array}{l}\text { Sensitivity }(95 \% \mathrm{Cl}) \\
\mathrm{n}=40\end{array}$ & $\begin{array}{l}\text { Specificity }(95 \% \mathrm{Cl}) \\
\mathrm{n}=101\end{array}$ \\
\hline \multirow[t]{3}{*}{ Serologically confirmed LD } & $\geq 4$-fold titre change in IFA & $38(24-53)$ & $100(96-100)$ \\
\hline & IgM and/or lgG seroconversion in ELISA & $30(18-45)$ & $99(95-100)$ \\
\hline & Combination of IFA and ELISA & $53(38-67)$ & $99(95-100)$ \\
\hline \multirow{3}{*}{$\begin{array}{l}\text { Serologically probable LD (single high or high } \\
\text { standing antibody level) }\end{array}$} & IFA-titre $\geq 128$ & $73(57-84)$ & $97(92-99)$ \\
\hline & ELISA IgM $\geq 120 \mathrm{U} / \mathrm{ml}$ and/or lgG $\geq 50 \mathrm{U} / \mathrm{ml}$ & $68(52-80)$ & $96(90-98)$ \\
\hline & Combination of IFA and ELISA & $78(63-88)$ & $96(90-98)$ \\
\hline \multirow[t]{3}{*}{ Serologically probable LD ( $\geq 1.5$ ratio in ELISA) } & IgM-ratio $\geq 1.5$ & $70(55-82)$ & $100(96-100)$ \\
\hline & IgG-ratio $\geq 1.5$ & $63(47-76)$ & $98(93-99)$ \\
\hline & IgM-ratio $\geq 1.5$ and/or IgG-ratio $\geq 1.5$ & $83(68-91)$ & $98(93-99)$ \\
\hline
\end{tabular}

LD: Legionnaires' disease. Data on sensitivity and specificity are in percent and based on 40 patients with confirmed LD from culture, PCR, and/or UAT and 101 non-cases (39 non-LD pneumonia patients and 62 healthy controls).

cases with corresponding values of 1.04 (IQR 1.00 1.14 ) and 1.09 (IQR $1.00-1.24$ ) for the non-cases. The highest combined sensitivity and specificity were found for a ratio of $>1.5$ in paired serum samples for both IgG and IgM (Table 1).

We found no statistically significant association between pneumonia severity score and sensitivity of the serological assays $(P>0.27)$ for any of the case definitions.

\section{Outbreak investigation}

During the initial outbreak investigation [16], 116 of the 225 patients referred to our hospital could not be classified definitely into LD or non-LD based on Legionella culture, PCR and/or UAT and epidemiological criteria (Figure 1). Following the evaluation study, these patients were studied by the serological assays using the three case-definitions as the specificity of more than $96 \%$ (Table 1) in an epidemic setting was considered acceptable.

Table 2 shows the results of all utilized tests. Fifty-six cases were previously diagnosed with LD by the acute phase tests [16], of which UAT was the most important. PCR and culture of sputum and lung tissue were positive in eight and ten cases, respectively, but the additive contribution of these tests was limited to four cases. However,

Table 2 Diagnostic tests for Legionnaires' disease in 103 outbreak patients

\begin{tabular}{|c|c|c|c|c|}
\hline Case definition & Laboratory test & $\begin{array}{l}\text { No. positive/ } \\
\text { no. tested (\%) }\end{array}$ & $\begin{array}{l}\text { Additional (cumulative) } \\
\text { no. of LD-cases }\end{array}$ & Comments \\
\hline \multirow{8}{*}{$\begin{array}{l}\text { Confirmed LD (direct demonstration } \\
\text { of Lp } 1 \text { infection) }\end{array}$} & Positive Lp 1 UAT* & $52 / 99(53)$ & 52 & \\
\hline & \multirow[t]{2}{*}{ Culture of Lp 1 (sputum)* } & \multirow[t]{2}{*}{$4 / 30(13)$} & \multirow[t]{2}{*}{$1(53)$} & Pos. UAT : 3 \\
\hline & & & & Neg UAT: 1 \\
\hline & \multirow{3}{*}{$\begin{array}{l}\text { Culture of Lp } 1 \\
\text { (lung tissue) }^{*}\end{array}$} & \multirow[t]{3}{*}{ 6/7 (86) } & \multirow[t]{3}{*}{$3(56)$} & Pos UAT: 3 \\
\hline & & & & Neg UAT: 2 \\
\hline & & & & UAT not done: 1 \\
\hline & \multirow[t]{2}{*}{ Positive PCR } & \multirow[t]{2}{*}{$8 / 25(32)$} & \multirow[t]{2}{*}{$0(56)$} & Sputum: 5/22 \\
\hline & & & & Lung tissue: $3 / 3$ \\
\hline \multirow[t]{2}{*}{ Confirmed LD** (serology) } & $\begin{array}{l}\geq 4 \text {-fold titre change } \\
\text { in IFA }\end{array}$ & $40 / 84(48)$ & $25(81)$ & \multirow[t]{2}{*}{$\begin{array}{l}13 \text { patients had } \geq 4 \text {-fold titre change } \\
\text { in IFA, but no seroconversion in ELISA. }\end{array}$} \\
\hline & $\begin{array}{l}\text { IgM/lgG seroconversion } \\
\text { in ELISA }\end{array}$ & $30 / 84(36)$ & $6(87)$ & \\
\hline \multirow{2}{*}{$\begin{array}{l}\text { Probable LD (single high or high } \\
\text { standing antibody level) }\end{array}$} & IFA-titre $\geq 128$ & $68 / 92(74)$ & $9(96)$ & \\
\hline & $\begin{array}{l}\text { ELISA } \operatorname{lgM} \geq 120 \mathrm{U} / \mathrm{ml} \\
\text { and/or } \operatorname{lgG} \geq 50 \mathrm{U} / \mathrm{ml}\end{array}$ & $59 / 92(64)$ & $1(97)$ & \\
\hline Probable LD (ELISA ratio $\geq 1.5$ ) & $\begin{array}{l}\text { ELISA IgM ratio } \geq 1.5 \\
\text { and/or IgG ratio } \geq 1.5\end{array}$ & 75/84 (89) & $6(103)$ & \\
\hline
\end{tabular}

LD: Legionnaires' disease, Lp 1: L. pneumophila serogroup 1, UAT: urinary antigen test. The proportion of serogroup 1 specificity to the outbreak strain on immunoblots among the serologically confirmed and probable LD cases was similar (70\%) to that of the culture, PCR, and/or UAT-confirmed LD cases. *Historical data [16]. Three of these patients were admitted to other hospitals in Norway and thus not shown in Figure 1.

**One of these cases was admitted to another hospital and thus not shown in Figure 1. 
isolation of Lp 1 was essential for the investigation of the source [16].

Serologically confirmed or probable LD was found in 47 patients (Table 2). Thirty-one of the cases had confirmed LD demonstrated by a $\geq 4$-fold titre change in IFA or seroconversion in ELISA. One of these, a local resident from the outbreak area, was admitted to another hospital and therefore not included in Figure 1. Ten cases had probable LD shown by a single high or high standing antibody titres. The alternative use of ELISA IgG/IgMratios, added only six cases to the total number. Thus, our data indicated that the outbreak comprised at least 103 cases.

LD was ruled out in 38 patients based on negative serology, whereas Legionella infection could not be verified or excluded in the remaining $32 \mathrm{CAP}$ patients (Figure 1). Twelve of these patients refused participation, seven had cognitive failure, ten died without microbiological sampling, and three had positive serology consistent with probable LD but died before Legionella exposure could be clarified, and neither UAT nor autopsy was performed.
The mean age for the $103 \mathrm{LD}$ cases was 67 years (range 35 - 94), and there were 64 males and $39 \mathrm{fe}-$ males. Ten of the cases, with a median age of 80 years (range 69 -94), died during hospitalisation (case fatality rate $10 \%)$. The demographic and serological results in Table 3 showed few significant differences between the two LD patient groups diagnosed by culture, PCR, and/ or UAT and serology, respectively. The latter group had less severe pneumonia, no deaths, and less frequent intensive care unit (ICU) admission than LD cases diagnosed in the acute phase. No statistically significant differences in antibody levels were observed between the two groups except for a higher IgM level at 3 months among cases with a positive culture/PCR/UAT.

Compared with all 94 non-LD patients (56 patients with confirmed non-LD and 38 patients with negative serology, Figure 1), LD cases had more severe pneumonia $(P=0.048)$ and a higher proportion of ICU admission $(P=0.019)$ than non-LD patients (Table 4$)$. However, chronic respiratory disease was more frequent in the non-LD group $(P=0.002)$. The group of 32 CAP patients

Table 3 Demographic and serological data among LD patients diagnosed by acute phase tests and serology

\begin{tabular}{|c|c|c|c|}
\hline & Positive culture/PCR/UAT $(n=56)$ & Positive serology only $(n=47)$ & $P$-value \\
\hline Age, mean years $\pm S D$ & $69 \pm 14$ & $65 \pm 12$ & 0.15 \\
\hline Male gender & $34(61)$ & $30(64)$ & 0.75 \\
\hline Chronic respiratory disease & $11(20)$ & $11(23)$ & 0.64 \\
\hline Active smoker & $22(39)$ & $17(36)$ & 0.69 \\
\hline Previously healthy & $20(36)$ & $18(38)$ & 0.79 \\
\hline Date of symptom onset & 16. May & 16. May & \\
\hline (interquartile range [IQR]) & (14.-19. May) & (14.-19. May) & 0.90 \\
\hline Inpatient treatment & $55(98)$ & $45(96)$ & 0.45 \\
\hline C-reactive protein $(\mathrm{mg} / \mathrm{L}) \pm \mathrm{SD}$ & $296 \pm 116$ & $237 \pm 89$ & 0.15 \\
\hline ICU-admission & $19(34)$ & $2(4)$ & $<0.001$ \\
\hline CRB-65 score $\geq 2$ & $33(59)$ & $16(34)$ & 0.012 \\
\hline Infiltrate $>$ one lobe & $13(23)$ & $9(19)$ & 0.83 \\
\hline Mortality & $10(18)$ & $0(0)$ & 0.002 \\
\hline IFA-titre, acute phase & $64(0-256)$ & $32(0-64)$ & 0.16 \\
\hline IFA-titre, after 1 month & $256(64-1024)$ & $128(128-256)$ & 0.11 \\
\hline IFA titre, after 3 months & $128(32-1024)$ & $64(32-128)$ & 0.15 \\
\hline ELISA IgG, acute phase & $41(10-115)$ & $20(9-52)$ & 0.25 \\
\hline ELISA IgG, after 1 month & $53(27-188)$ & $66(30-193)$ & 0.96 \\
\hline ELISA IgG, after 3 months & $60(17-312)$ & $33(12-131)$ & 0.20 \\
\hline ELISA IgM, acute phase & $24(4-93)$ & $8(3-150)$ & 0.15 \\
\hline ELISA IgM, after 1 month & $47(22-195)$ & $29(16-87)$ & 0.11 \\
\hline ELISA IgM, after 3 months & $21(10-76)$ & $11(6-30)$ & 0.04 \\
\hline
\end{tabular}

Demographic data are no. (\%) of patients unless otherwise indicated. Serological results are given in median (interquartile range) titre for IFA and U/mI for ELISA $\lg G$ and $\lg M$.

ICU: Intensive care unit. CRB-65 score $\geq 2$ indicates moderate to severe pneumonia. 
Table 4 Demography of different patient groups referred to the hospital during the outbreak

\begin{tabular}{|c|c|c|c|}
\hline & Legionnaires' disease $(n=103)$ & CAP, non-Legionella $(\mathrm{n}=94)$ & CAP of uncertain Legionella status $(n=32)$ \\
\hline Age, mean years $\pm S D$ & $67 \pm 13$ & $65 \pm 17$ & $75 \pm 16$ \\
\hline Male gender & $64(62)$ & $50(53)$ & $15(47)$ \\
\hline Active smoker & $39(38)$ & $28(30)$ & $6(19)$ \\
\hline Chronic respiratory disease $^{1}$ & $22(21)$ & $39(41)$ & $12(38)$ \\
\hline Diabetes & $16(16)$ & $12(13)$ & $3(9)$ \\
\hline Previously healthy & $38(37)$ & $26(28)$ & $6(19)$ \\
\hline Inpatient treatment & $100(97)$ & $85(90)$ & $29(91)$ \\
\hline ICU-admission ${ }^{2}$ & $21(20)$ & $8(9)$ & $4(13)$ \\
\hline CRB-65 score $\geq 2^{3}$ & $48(47)$ & $28(30)$ & $15(47)$ \\
\hline Infiltrate > one lobe & $23(22)$ & $12(13)$ & $3(9)$ \\
\hline Mortality & $10(10)$ & $6(6)$ & $6(19)$ \\
\hline
\end{tabular}

${ }^{1} P=0.002$.

${ }^{2} P=0.019$.

${ }^{3} P=0.048$.

Patients with Legionnaires' disease, community-acquired pneumonia (CAP) of non-LD aetiology and CAP of uncertain Legionella status referred to hospital from 9 May to 12 June are shown. Data are no. (\%) of patients unless otherwise indicated. $P$-values in footnotes indicate the comparison between Legionnaires' disease and CAP of non-Legionella aetiology.

with uncertain Legionella status had a high mean age and mortality, but statistical comparisons of this group with the two others are less reliable due to its heterogeneity as described above. Figure 2 demonstrates the hospitalisation date for patients with LD diagnosed by culture/PCR/UAT and serology, respectively, non-LD, and CAP of unknown aetiology during the defined five-week period. The number of admissions for LD cases mirrored the outbreak epidemic curve [16]. The non-LD admission curve, which was expected to fluctuate around a mean of 2.3 per day based on hospitalization rates in 2001 - 2004, also peaked, but two days after the outbreak alert. This curve indicated an over-referral of approximately 15 non-LD patients probably caused by the media attention following the outbreak. The 32 patients with CAP of unknown aetiology were scattered throughout the period with a peak two days before the outbreak alert.

\section{Lp 1 specificity of LD case sera}

Immunoblotting was performed to test whether the antibody responses observed with the polyvalent ELISA and IFA were directed to the serogroup-specific LPS of the Lp 1 outbreak strain. Sera from 44 culture/UAT-positive and 45 UAT-negative/serology positive LD cases were available for immunoblotting. Figure 3 shows the IgG and IgM responses with the outbreak strain for 25 randomly chosen UAT-negative cases. Antibody binding to the LPS region below $25 \mathrm{kDa}$ dominated in addition to individual antibody reactions in the 25 to $80 \mathrm{kDa}$

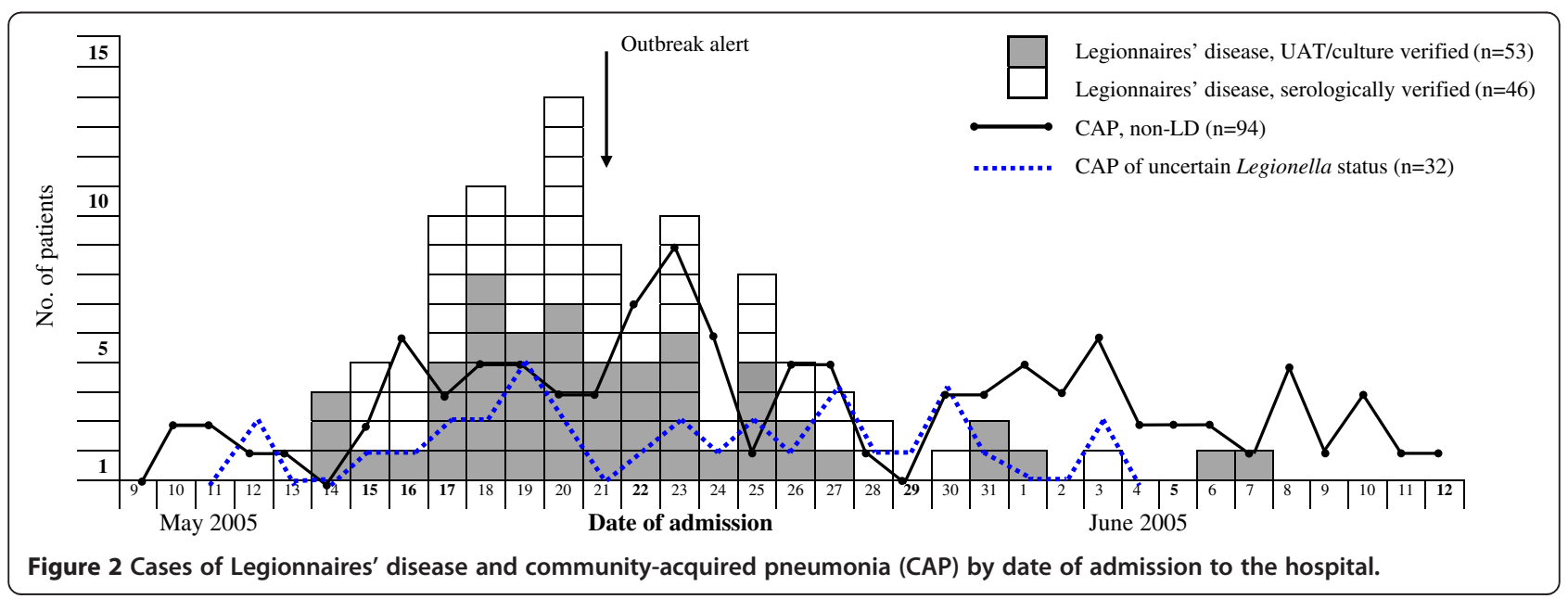




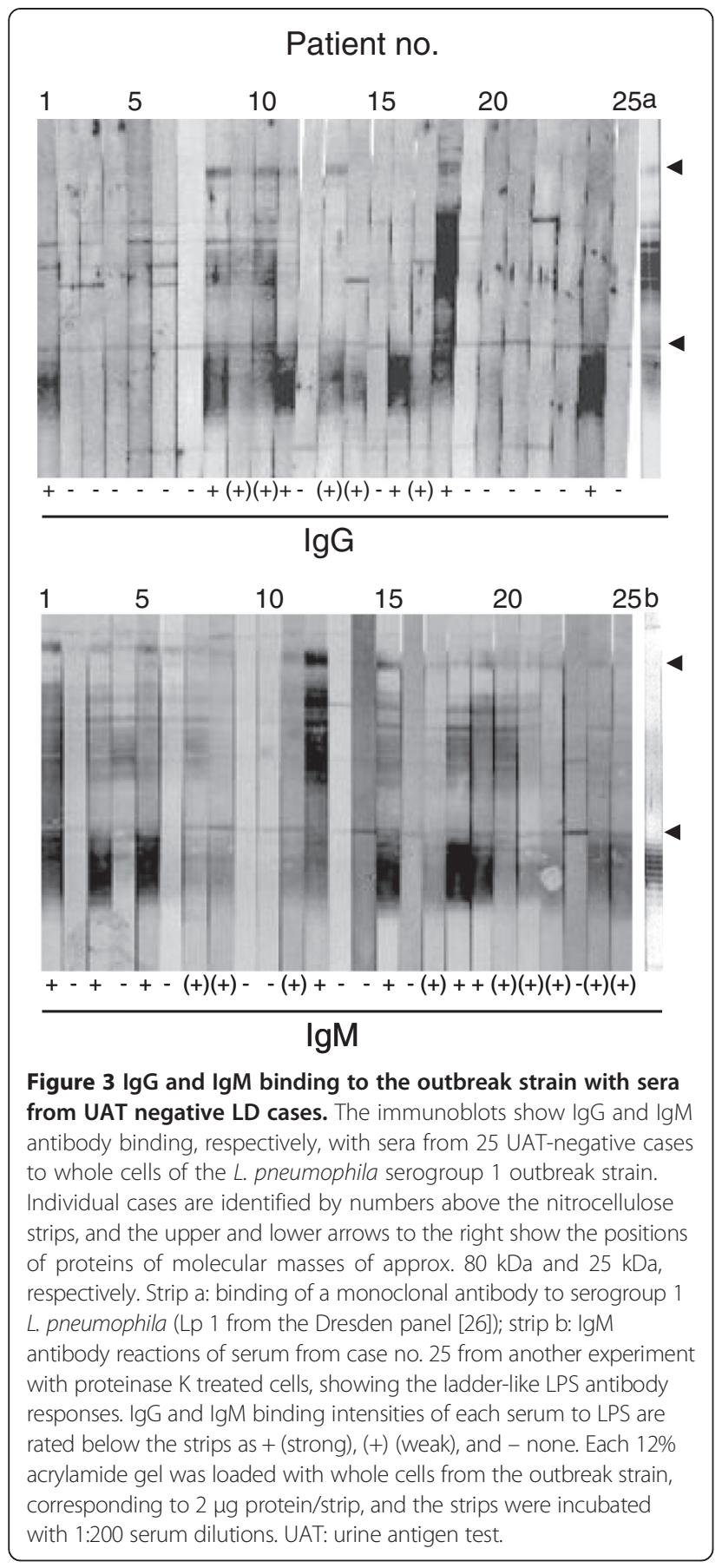

molecular mass range. For IgM in particular, the binding intensities to the LPS region below $25 \mathrm{kDa}$ corresponded with intensities of a smear in the higher molecular weight range. Among all 45 UAT-negative cases, 30 (67\%) had IgG and/or IgM antibodies that showed strong (16 cases) or weak binding (14 cases) to LPS on the blots. A similar proportion of the UAT-positive cases/(30/44; 68\%) also showed corresponding LPS bands (19 and 11 cases with strong and weak bands, respectively), whereas no such reactions were observed with sera from the 39 confirmed
non-LD patients (data not shown). LPS band intensities generally corresponded to the IgG or IgM antibody levels in ELISA. Proteinase K treatment of the outbreak strain gave similar LPS responses as the untreated cells, but the distinct high molecular weight bands were absent and the ladder-like LPS pattern below $25 \mathrm{kDa}$ became more distinct after proteolysis (Figure 3; strip b). In comparison with these LPS responses, 15 (33\%) and 26 (59\%) of the sera from the UAT-negative and UAT-positive cases, respectively, demonstrated strong or weak LPS bands with a proteolytically treated serogroup 1 France/Allentown isolate (data not shown). Thus, more UAT-positive cases seemed to have cross-reactive antibodies to this strain than the UAT-negative cases.

\section{Discussion}

This Legionella outbreak demonstrated the challenges of the microbiological diagnosis of LD: 1) the acute phase tests (culture, PCR, and UAT) underestimated the number of cases; 2) the traditional serological criterion of a $\geq 4$-fold titre increase yielded poor sensitivity in this outbreak population; and 3) even when all diagnostic tests were employed, there was still an unexplained excess of hospitalized CAP patients in whom Legionella infection could not be verified or excluded indicating shortcomings of the microbiological tools. Previously, the acute phase tests diagnosed 56 LD outbreak cases [16]. Our study identified an additional 47 cases with the serological assays, thus the outbreak comprised a total of 103 LD cases, of whom 87 were confirmed and 16 were probable cases. Another group of 32 patients with CAP of high mortality may also belong to the outbreak.

UAT has emerged as the most common test for laboratory diagnosis of LD [2] because of its simplicity and high specificity. Although it was useful during the acute phase, UAT was positive in only 52 of the 99 cases (53\%) who were tested (Table 2). This number corresponded to those from two other outbreaks $[28,29]$ and a recent methodological study [30]. However, a metaanalysis [14] reported a sensitivity of $74 \%$, and in infections caused by Lp 1 strains with the virulence-associated epitope, recognized by the $3 / 1$ monoclonal antibody [26] including the outbreak strain [15], the sensitivity was more than $90 \%$ [13]. As UAT has lower sensitivity in mild LD [12], the sensitivity discrepancy is probably due to the broader clinical spectrum including less severe CAP in the outbreak population. Besides UAT, only four more cases were identified by culture of sputum and lung tissue and none by PCR, but fewer samples were analysed by these two assays (Table 2).

Evaluation of the serological assays (Table 1) demonstrated lower sensitivity than Yzerman et al. [18] reported with LD patients from the large Dutch outbreak with the 
same assays, especially for the $\geq 4$-fold IFA-titre rise (38\% vs. $61 \%$ ) and ELISA seroconversion (30\% vs. $64 \%$ ). However, the results were more similar for the high standing titre definition ( $73 \%$ vs. $86 \%$ in IFA and $68 \%$ vs. $75 \%$ in ELISA). Early sampling may lead to higher sensitivity; in our study, the mean time from disease onset to sampling of the acute-phase sera was 11 days (range 5 - 15) compared with 8 days (range $0-15$ ) in the Dutch study [18]. This suggested that seroconversion may already have occurred, especially for IgM antibodies [31]. The use of paired sera from LD cases in the evaluation study (Table 1) further indicated the presence of exclusion bias as the most severely ill LD cases died before convalescent sera could be sampled. Thus, the low mortality among the LD cases in our evaluation study ( $0 \%$ vs. $9 \%$ in the Dutch study), indicating differences in illness severity, and the different Lp 1 strains in the two outbreaks [18] may present alternative explanations for the divergent results. Our study did not demonstrate any association between the sensitivity of the serological tests and pneumonia severity, but the statistical power was low.

The alternative ELISA evaluation, based on the ratios in paired samples of $\operatorname{IgG}$ and $\operatorname{Ig} M \geq 1.5$, respectively, showed the highest combined sensitivity and specificity of the three evaluated case-definitions (Table 1). The higher sensitivity was obtained because several cases with high ratios were negative according to the manufacturer's cut-off. A similar method has been validated in ELISA for infections caused by Mycoplasma pneumonia and Chlamydophila pneumonia [32,33], but to our knowledge, this method, which detected six additional LD cases (Table 2), has not previously been applied for Legionella testing. As about 20-30\% of LD patients do not develop significantly increased antibody levels even after prolonged observation [34], the advantage of this method is to ignore an absolute cut-off level which must be set high in order to produce a low level of false positives. The challenge remains to find a reasonable cut-off to avoid clinically irrelevant changes in the low antibody range, and the specificity will probably suffer in a non-outbreak study.

Selection of non-cases for evaluation of the specificity was not optimal. Ideally, this group should consist of non-LD CAP patients only, but misclassification was difficult to avoid because of the epidemiological situation and the low negative predictive value of a negative UAT. We therefore included healthy controls to increase the power of the specificity analysis. Although this group was also exposed to the Legionella outbreak, as demonstrated by the slightly higher antibody levels among healthy blood donors in the outbreak county compared with donors in a non-exposed county [23], it differed from the CAP population in both age and past medical history which might have affected the results. There was no seroconversion in the healthy control group, and the antibody levels (median IgG $9 \mathrm{U} / \mathrm{ml}$, range $1-111$ $\mathrm{U} / \mathrm{ml}$, and median IgM $14 \mathrm{U} / \mathrm{ml}$, range $2-82 \mathrm{U} / \mathrm{ml}$ ) were comparable to those demonstrated one year after the outbreak in healthy blood donors from the same county [23].

As the polyvalent antigens in the ELISA and IFA may affect the case definitions, immunoblotting with the LP 1 outbreak strain was performed to investigate if the antibody responses were directed to the serogroupspecific LPS antigen of this strain. These experiments showed that two-thirds of both the UAT-positive and the UAT-negative LD cases had IgG and/or IgM antibodies that reacted with the LPS. About the same proportion of the UAT-positive cases, but only $30 \%$ of the UAT-negative cases showed cross-reactive antibodies with LPS of a subgroup France/Allentown Lp 1 strain that also carry the virulence-associated monoclonal $3 / 1$ epitope [26]. It is less likely that this cross-reaction is caused by infection of France/Allentown strains as these are rarely seen in Norway after 2001 [15]. Some crossreactions may probably occur as various subgroups of Lp 1 strains show the same ladder-like profiles in silverstained gels [35]. A rabbit serum to one Lp1 subgroup was found to cross-react with the other subgroups on immunoblots, but a corresponding reaction was only observed with a few of the 14 serogroups of L. pneumophila $[35,36]$. In Norway, the incidence of LD is low, and only a small number of seroresponders (2.3\%) among healthy blood donors was observed in the outbreak county [23]. Together with the same antibody levels (Table 3) in the UAT positive and negative cases, the same LPS-specific antibody responses, the same time period, and home addresses, our findings suggested that the UAT-negative LD cases were most likely infected with the outbreak strain and not with other L. pneumophila strains.

This LD outbreak probably included more than the 103 verified cases. The average number of patients referred to our hospital with CAP during the same fiveweek period in the preceding four years was 80 (range 72-86). Comparison of this number with the 225 CAP patients admitted during the outbreak in 2005 (Figure 1), indicated an excess of 145 patients and possible LD cases. This observation may be supported by another outbreak study which suggested that UAT and culture detected less than $40 \%$ of the LD patients, when the unexplained excess of CAP patients with possible LD was taken into consideration [29]. Based on a similar assumption for this outbreak (56 cases originally detected $=40 \%$ of the outbreak), we might expect to find about 140 patients with LD. A major limitation of these calculations was the uncertainty of increased referral because of the outbreak alert. However, no more than 15 
patients could be attributed to this effect as indicated by Figure 2. Compared with the previous four years, the proportion of outpatient treatment was approximately the same indicating comparable referral and admission strategies. Furthermore, in 32 hospitalized CAP patients with high mortality, Legionella infection could not be ruled out based on epidemiological or laboratory criteria. A considerable proportion of these was admitted during the main bulk of referrals immediately before the outbreak was recognized (Figure 2). This finding suggested that the incidence is underestimated even in prospective studies. Underdiagnosis of non-severe LD might not seem important as the mortality is low. However, recovery from LD is poorly studied, but might include reduced pulmonary function [37,38], fatigue, and even posttraumatic stress disorder [39].

\section{Conclusions}

Our study demonstrated that serological testing is a valuable tool to determine the total number of patients during an LD outbreak investigation. The serological assays detected 47 more cases than the 56 cases previously diagnosed by culture, PCR, and/or UAT. To our knowledge, the Legionella outbreak in 2005 in Norway is the largest outbreak reported until now in the Nordic countries.

\section{Competing interests}

The authors declare that they have no competing interests.

\section{Authors' contributions}

$\varnothing S$ and JR conceived and designed the study, collected and analysed the clinical data, and drafted the manuscript. EW was responsible for interpretation of the ELISA and immunoblotting data, participated in the design of the study, and in drafting and revising the manuscript. ISA participated in the design and in interpretation of the ELISA and IFA data and in revising the manuscript. AK and ER participated in the design and interpretation of the IFA studies, the non-serological microbiological analyses, and in revising the manuscript. KB performed the ELISA and immunoblotting studies and participated in revising the manuscript. All authors read and approved the final manuscript.

\section{Acknowledgements}

We thank Elisabeth Fritzsønn, Norwegian Institute of Public Health, for valuable technical support in establishing the ELISA test, Eva H. Lindberg for performing the IFA, Randi Bruaset and the staff at the Medical Department, all at Østfold Hospital Trust, for recruiting patients and healthy controls, and Leiv Sandvik, Oslo University Hospital, for helpful statistical advice. The study was financially supported by a grant from the South-Eastern Norway Regional Health Authority.

Parts of this work were presented at Legionella 2009, Paris, France. Ref: Wedege, E, Bolstad, K, Kanestrøm, A, Lindberg E. H. and Simonsen $\varnothing$. Comparative immunological studies of sera from patients hospitalized during a large outbreak of Legionnaires' Disease, abstr. P80. Legionella 2009, Paris, France.

\section{Author details}

${ }^{1}$ Department of Medicine, Østfold Hospital Trust, Fredrikstad, Norway. ${ }^{2}$ Department of Bacteriology and Immunology, Division of Infectious Disease Control, Norwegian Institute of Public Health, Oslo, Norway. ${ }^{3}$ Department of Clinical Microbiology, Østfold Hospital Trust, Fredrikstad, Norway.
Received: 9 July 2014 Accepted: 13 March 2015

Published online: 28 March 2015

\section{References}

1. Norwegian Surveillance System for Communicable Diseases (MSIS). [http://www.msis.no]

2. Ricketts KD, Joseph CA, European Working Group for Legionella Infections. Legionnaires disease' in Europe: 2005-2006. Euro Surveill. 2007;12:E7-8.

3. Garasen H, Sagvik E, Kvendbo JF, Lian A, Jacobsen T, Nylenna M. Legionella in Trondheim, Norway - determining sources of contagion and tracing risk environments. Tidsskr Nor Laegeforen. 2005;125:1791-3.

4. Marston BJ, Plouffe JF, File Jr TM, Hackman BA, Salstrom SJ, Lipman HB, et al. Incidence of community-acquired pneumonia requiring hospitalization. Results of a population-based active surveillance Study in Ohio. The Community-Based Pneumonia Incidence Study Group. Arch Intern Med. 1997;157:1709-18.

5. Bohte R, van Furth $R$, van den Broek PJ. Aetiology of community-acquired pneumonia: a prospective study among adults requiring admission to hospital. Thorax. 1995;50:543-7.

6. von Baum H, Ewig S, Marre R, Suttorp N, Gonschior S, Welte T, et al. Community-acquired Legionella pneumonia: new insights from the German competence network for community-acquired pneumonia. Clin Infect Dis. 2008;46:1356-64

7. Macfarlane JT, Finch RG, Ward MJ, Macrae AD. Hospital study of adult community-acquired pneumonia. Lancet. 1982;320:255-8.

8. Benin AL, Benson RF, Besser RE. Trends in Legionnaires disease, 1980-1998: declining mortality and new patterns of diagnosis. Clin Infect Dis. 2002;35:1039-46.

9. Yu VL, Plouffe JF, Pastoris MC, Stout JE, Schousboe M, Widmer A, et al. Distribution of Legionella species and serogroups isolated by culture in patients with sporadic community-acquired legionellosis: an international collaborative survey. J Infect Dis. 2002;186:127-8.

10. Maurin M, Hammer L, Gestin B, Timsit JF, Rogeaux O, Delavena F, et al. Quantitative real-time PCR tests for diagnostic and prognostic purposes in cases of legionellosis. Clin Microbiol Infect. 2010;16:379-84.

11. Carratalà J, Garcia-Vidal C. An update on Legionella. Curr Opin Infect Dis. 2010;23:152-7.

12. Yzerman EP, den Boer JW, Lettinga KD, Schellekens J, Dankert J, Peeters M. Sensitivity of three urinary antigen tests associated with clinical severity in a large outbreak of Legionnaires' disease in The Netherlands. J Clin Microbiol. 2002:40:3232-6.

13. Helbig JH, Uldum SA, Bernander S, Luck PC, Wewalka G, Abraham B, et al. Clinical utility of urinary antigen detection for diagnosis of communityacquired, travel-associated, and nosocomial Legionnaires' disease. J Clin Microbiol. 2003;41:838-40.

14. Shimada T, Noguchi Y, Jackson JL, Miyashita J, Hayashino Y, Kamiya T, et al. Systematic review and metaanalysis: urinary antigen tests for Legionellosis. Chest. 2009;136:1576-85.

15. Wedege E, Bolstad K, Borgen K, Fritzsonn E, Caugant DA. Molecular characterization of clinical and environmental isolates of Legionella pneumophila in Norway, 2001-2008. Scand J Infect Dis. 2013;45:59-64.

16. Nygard K, Werner-Johansen O, Ronsen S, Caugant DA, Simonsen O, Kanestrom A, et al. An outbreak of Legionnaires disease caused by longdistance spread from an industrial air scrubber in Sarpsborg, Norway. Clin Infect Dis. 2008;46:61-9.

17. Olsen JS, Aarskaug T, Thrane I, Pourcel C, Ask E, Johansen G, et al. Alternative routes for dissemination of Legionella pneumophila causing three outbreaks in Norway. Environ Sci Technol. 2010;44:8712-7.

18. Yzerman EP, den Boer JW, Lettinga KD, Schel AJ, Schellekens J, Peeters M. Sensitivity of three serum antibody tests in a large outbreak of Legionnaires' disease in the Netherlands. J Med Microbiol. 2006;55:561-6.

19. Virion\Serion: Serion ELISA classic. Legionella pneumophila 1-7 lgG/lgM. Instructions. [http://www.virion-serion.de/download/gebrauchsanweisung/ legionella_pneumophila/IFU-Legionella-EN-ES-GR-PT-CZ.pdf]

20. European Centre for Disease Prevention and Control: EU case definition. [http://ecdc.europa.eu/en/activities/surveillance/ELDSNet/Pages/EU\%20case \%20definition.aspx]

21. Lim WS, van der Eerden MM, Laing R, Boersma WG, Karalus N, Town Gl, et al. Defining community-acquired pneumonia severity on presentation to hospital: an international derivation and validation study. Thorax. 2003;58:377-82. 
22. Ciesielski CA, Blaser MJ, Wang WL. Serogroup specificity of Legionella pneumophila is related to lipopolysaccharide characteristics. Infect Immun. 1986;51:397-404.

23. Wedege E, Bergdal T, Bolstad K, Caugant DA, Efskind J, Heier HE, et al. Seroepidemiological study after a long-distance industrial outbreak of Legionnaires' disease. Clin Vaccine Immunol. 2009;16:528-34.

24. Hitchcock PJ, Brown TM. Morphological heterogeneity among Salmonella lipopolysaccharide chemotypes in silver-stained polyacrylamide gels. J Bacteriol. 1983;154:269-77.

25. Tsai CM, Frasch CE. A sensitive silver stain for detecting lipopolysaccharides in polyacrylamide gels. Anal Biochem. 1982;119:115-9.

26. Helbig JH, Bernander S, Castellani Pastoris M, Etienne J, Gaia V, Lauwers $\mathrm{S}$, et al. Pan-European study on culture-proven Legionnaires' disease: distribution of Legionella pneumophila serogroups and monoclonal subgroups. Eur J Clin Microbiol Infect Dis. 2002;21:710-6.

27. Open Source Epidemiologic Statistics for Public Health, Version 2.3. [http://www.openepi.com]

28. Rojas A, Navarro MD, Fornés FE, Serra E, Simarro E, Rojas J, et al. Value of serological testing for diagnosis of legionellosis in outbreak patients. J Clin Microbiol. 2005;43:4022-5.

29. Hugosson A, Hjorth M, Bernander S, Claesson BE, Johansson A, Larsson H, et al. A community outbreak of Legionnaires' disease from an industrial cooling tower: assessment of clinical features and diagnostic procedures. Scand J Infect Dis. 2007;39:217-24.

30. Svarrer CW, Luck C, Elverdal PL, Uldum SA. Immunochromatic kits Xpect Legionella and BinaxNOW Legionella for detection of Legionella pneumophila urinary antigen have low sensitivities for the diagnosis of Legionnaires' disease. J Med Microbiol. 2012;61:213-7.

31. Kallings I, Nordstrom K. The pattern of immunoglobulins with special reference to IgM in Legionnaires' disease patients during a 2 year follow-up period. Zentralbl Bakteriol Mikrobiol Hyg A. 1983;255:27-32.

32. De Ory F, Guisasola ME, Eiros JM. Detection of Chlamydophila pneumoniae lgG in paired serum samples: comparison of serological techniques in pneumonia cases. APMIS. 2006;114:279-84.

33. Talkington DF, Shott S, Fallon MT, Schwartz SB, Thacker WL. Analysis of eight commercial enzyme immunoassay tests for detection of antibodies to Mycoplasma pneumoniae in human serum. Clin Diagn Lab Immunol. 2004;11:862-7.

34. Lück P, Helbig J, Schuppler M. Epidemiology and laboratory diagnosis of Legionella infections. J Lab Med. 2002;26:174-82.

35. Jurgens D, Fehrenbach FJ. Cross-reacting lipopolysaccharide antigens in Legionella pneumophila serogroups 1 to 14. Infect Immun. 1995;63:2180-4.

36. Nolte FS, Conlin CA. Major outer membrane protein of Legionella pneumophila carries a species-specific epitope. J Clin Microbiol. 1986;23:643-6.

37. Lattimer GL, Rhodes 3rd LV, Salventi JS, Galgon JP, Stonebraker V, Boley S, et al. The Philadelphia epidemic of Legionnaire's disease: clinical, pulmonary, and serologic findings two years later. Ann Intern Med. 1979;90:522-6.

38. Jonkers RE, Lettinga KD, Pels Rijcken $T H$, Prins JM, Roos CM, van Delden OM, et al. Abnormal radiological findings and a decreased carbon monoxide transfer factor can persist long after the acute phase of Legionella pneumophila pneumonia. Clin Infect Dis. 2004;38:605-11.

39. Lettinga KD, Verbon A, Nieuwkerk PT, Jonkers RE, Gersons BP, Prins JM, et al. Health-related quality of life and posttraumatic stress disorder among survivors of an outbreak of Legionnaires disease. Clin Infect Dis. 2002;35:11-7.

\section{Submit your next manuscript to BioMed Central and take full advantage of:}

- Convenient online submission

- Thorough peer review

- No space constraints or color figure charges

- Immediate publication on acceptance

- Inclusion in PubMed, CAS, Scopus and Google Scholar

- Research which is freely available for redistribution 